\title{
Outcome after prenatal diagnosis of the hypoplastic left heart syndrome
}

\author{
L D Allan, H D Apfel, B F Printz
}

\begin{abstract}
Objective-To derive accurate survival figures in the current surgical era for counselling in early pregnancy after the diagnosis of fetal hypoplastic left heart syndrome.

Setting-A tertiary referral centre for paediatric cardiology and cardiac surgery. Design-A retrospective study of the outcome in all cases of hypoplastic left heart syndrome presenting in fetal life between mid-1993 and the end of 1996.
\end{abstract}

Patients-The diagnosis was made in $\mathbf{3 0}$ fetuses. In four of 12 identified before 24 weeks' gestation the mothers chose to terminate the pregnancy. There was an intention to treat in 24 of the remaining fetuses.

Main outcome measure-Survival to six months of postnatal life.

Results-Of the 24 infants, five were not offered Norwood stage 1 because of trisomy $18(n=2)$, unfavourable cardiac anatomy $(n=2)$, or neurological impairment $(n=1)$. One further infant did not survive to cardiac surgery after gastrointestinal surgery. Of the remaining 18 patients, eight had features that were considered to increase the risk of surgical repair. Of the 18 patients who underwent Norwood stage 1 , there were nine survivors. There was a survival rate of $70 \%$ in infants undergoing surgery with no complicating features, a $50 \%$ survival of the all surgical candidates, and $37.5 \%$ survival from an intention to treat position.

Conclusions-At the initial diagnosis of fetal hypoplastic left heart syndrome, the overall survival appears to be less than $40 \%$. Evaluation must include detailed extracardiac and intracardiac assessment to predict the risks of surgical treatment. Prenatal counselling can be modified as pregnancy advances, depending on the detection or exclusion of complicating factors.

(Heart 1998;79:371-374)

Pediatric Cardiology, Babies Hospital 2N, Columbia

Presbyterian Medical Center, 3959

Broadway, New York, NY 10032, USA

L D Allan

H D Apfel

E F Printz

Correspondence to: Dr Allan.

email: la48@columbia.edu

Accepted for publication 15 December 1997

Keywords: fetus; congenital heart disease; hypoplastic left heart syndrome

The hypoplastic left heart syndrome is usually readily detectable before 20 weeks' gestation by echocardiographic examination of the four chamber view of the fetal heart, and is one of the most common abnormalities detected in the fetus. ${ }^{1}$ Much more rarely, it can evolve in later pregnancy from critical aortic stenosis. ${ }^{2}$ Prenatal diagnosis of the hypoplastic left heart syndrome has led to a decreasing prevalence of this syndrome in infancy ${ }^{3}$ as a result of termination of pregnancy, particularly before treatment options became generally available. ${ }^{4}$ As the results of the Norwood staged palliation for the hypoplastic left heart syndrome have improved, however, mothers increasingly elect to continue with an affected pregnancy. In order to counsel parents accurately in early pregnancy, it is essential to understand the natural history of this condition and to be able to describe the likely survival after surgery in the current era. As our surgical results are comparable with those reported in other series, ${ }^{5-7}$ we analysed the outcome in a recent series of patients where the diagnosis of the hypoplastic left heart syndrome was made in utero.

\section{Methods}

Of a series of over 160 fetal cardiac anomalies detected in our institution between June 1993 and the end of 1996, 30 were examples of critical mitral stenosis or mitral atresia with aortic concordance. The aorta was atretic with reverse flow in the transverse arch in 27 cases, and patent in three cases, with an associated ventricular septal defect. The gestational age at presentation to the referral centre ranged between 17 and 41 weeks' gestation, with a mean and median of 29 weeks. Four sets of parents chose to terminate the pregnancy and two chose not to have the infant treated surgically after delivery. The remaining 24 fetuses, where there was an intention to treat, form the focus of this study. In all continuing pregnancies an attempt was made to assess suitability for Norwood stage 1 . This involved fetal karyotyping and a detailed fetal anatomical survey. In addition, the following cardiac structures were evaluated: the pulmonary veins by colour and pulsed Doppler; the site and size of the atrial septal defect; the morphology of the tricuspid and pulmonary valves; qualitative right ventricular function. The outcome after delivery was studied retrospectively.

\section{Results}

Two fetuses proved to have trisomy 18 at amniocentesis. These neonates were not offered surgical treatment. A further fetus had Turner's syndrome and was mosaic for a deletion of chromosome 8 but in view of uncertainty about the future severity of neurological impairment, the parents opted for surgical treatment. Two fetuses proved to have extracardiac malformations - a tracheo-oesophageal fistula and multiple small bowel atresias, respectively. Both patients had early gastrointestinal surgery. The first patient did not 

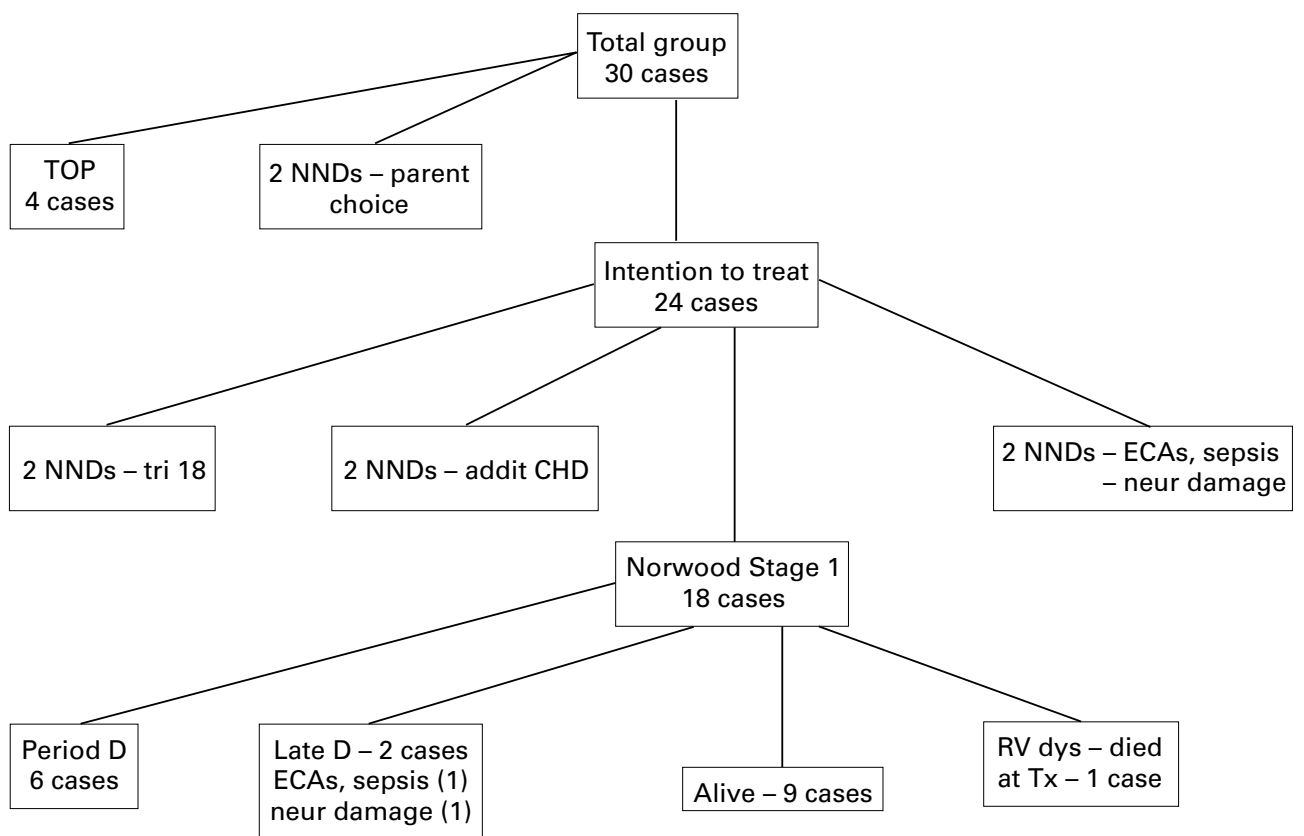

Figure 1 Outcome of 30 cases of fetal hypoplastic left heart syndrome. CHD, congenital heart disease; D, death; ECAs, extracardiac abnormalities; NND, neonatal death; neur; neurological; RV dys, right ventricular dysfunction; TOP, termination of pregnancy; tri 18, trisomy 18; Tx, transplantation.

survive to undergo the Norwood stage 1 procedure but died of sepsis postoperatively. One further patient suffered a cerebrovascular accident in the first days of life with resulting severe neurological impairment. This neonate was not offered surgery.

\section{ADDITIONAL INTRACARDIAC ANOMALIES}

Two neonates were not considered suitable for Norwood stage 1 because of unfavourable additional cardiac findings. These were tricuspid valve dysplasia with moderate regurgitation in one case and significant pulmonary stenosis in the other. In a further case, there was an intact atrial septum with normal pulmonary venous connection but supracardiac total anomalous pulmonary venous return; five additional cases had evidence of a restrictive foramen ovale on examination of the pulmonary venous flow profile. None of the patients in our series had right ventricular dysfunction prenatally.

SURGICAL OUTCOME

Eighteen infants underwent Norwood stage 1, 17 in our centre. Six died within 24 hours of surgery, one being preterm, weighing $1.8 \mathrm{~kg}$. The patient with multiple bowel atresias died from sepsis and right ventricular dysfunction four months after Norwood stage 1. The patient managed surgically at another centre had severe neurological impairment and died. One survivor of stage 1 Norwood had severe right ventricular dysfunction, was listed for heart transplantation, and died during this

Table 1 Potential hazards between diagnosis and surgery for the fetus with hypoplastic left heart syndrome

Chromosomal anomaly

Additional cardiac malformations

Extracardiac malformations

Prematurity

Preoperative neurological damage procedure. There were 10 neonates with no complicating features at surgery, of whom seven survived. The outcome for the total group of 30 pregnancies is summarised in fig 1 .

\section{Discussion}

Although there is no universal agreement on the usefulness of routine ultrasound during pregnancy, most pregnant women have at least one scan. Examination of the heart using a four chamber view constitutes part of the fetal evaluation recommended by the bodies that supervise prenatal ultrasound, such as the American Institute of Ultrasound in Medicine and the American College of Obstetrics and Gynecology. Although it is possible to detect nearly every four chamber view anomaly during routine obstetric screening, ${ }^{8}$ in practice many cardiac defects are still overlooked. ${ }^{9-11}$ Montana et al, in a recent publication, showed that overall only $28 \%$ of cases of hypoplastic left heart syndrome were detected prenatally, although the rate of detection had improved between 1990 and 1994 from $18 \%$ to $40 \% .^{11}$ This is comparable with our current experience-over a three and half year period, 60 patients presented to our institution with the hypoplastic left heart syndrome, of whom $30(50 \%)$ were diagnosed prenatally.

Of the 30 cases, 12 were diagnosed before 24 weeks' gestation. Four sets of parents chose to interrupt the pregnancy. The low rate of termination of pregnancy $(33 \%$ of those diagnosed early) and "compassionate care" choice (two of 26 continuing pregnancies) indicate that the patients who come to our centre are selected and in general are seeking further information about surgical treatment after initial diagnosis and counselling elsewhere.

When counselling parents in early pregnancy about the results of palliative surgery, it is important to understand that there are several 
"hazards" the fetus faces before reaching surgery (table 1).

The first hazard is the fetal karyotype. There were chromosomal anomalies in three of 30 cases $(10 \%)$. This is higher than that found in a previous series of 161 cases, ${ }^{1}$ where there was a rate of $4 \%$, but the numbers in this present series are small. It is therefore important to karyotype all ongoing pregnancies with the diagnosis of the hypoplastic left heart syndrome before rearranging the perinatal care.

The second hazard is the finding of extracardiac malformations. The complex nature of the first surgery in the hypoplastic left heart syndrome is such that additional problems will have an impact on the survival rate. There were extracardiac malformations in two cases in our series that contributed significantly to the fatal outcome, one before and the other after Norwood stage 1 . Both were gastrointestinal anomalies that did not become evident in either case until the third trimester of pregnancy, despite regular and skilled sonographic surveillance.

An additional hazard is prematurity, which occurred in one case. Prematurity and the weight of $1.8 \mathrm{~kg}$ at surgery was thought to contribute to perioperative death.

Prenatal diagnosis and delivery within a cardiac centre might be expected to give the best results for postnatal treatment, ${ }^{12}$ by avoiding the hazards of neonatal transfer and the possibility of hypoxia or acidosis causing neurological damage. However, one neonatedespite delivery at the cardiac centre and ideal perinatal support-was found to be neurologically impaired before surgery owing to cerebral thrombosis of unknown origin.

Eight fetuses had other intracardiac findings that influenced the management or postnatal outcome. One fetus had significant tricuspid dysplasia with moderate regurgitation and one had significant pulmonary valve stenosis. Both these neonates were considered unsuitable for Norwood stage 1. A restrictive or intact atrial septum in the setting of a hypoplastic left heart syndrome has been associated with a poor survival. ${ }^{13}$ Prenatal detection in six fetuses allowed early intervention, with survival in two of the five in whom surgery was offered. The restrictive nature of the atrial septum was thought to contribute to death in one of the three who died. Identification of cases where the intracardiac findings are unfavourable for Norwood palliation could have allowed rerouting to neonatal transplantation, ${ }^{14}$ although this was not considered in our cases by parental choice.

CONCLUSIONS

Of 24 cases of the hypoplastic left heart syndrome diagnosed prenatally where there was an intention to treat after delivery, 18 cases reached surgery and nine survived. This represents $37.5 \%$ survival in those cases where there was an intention to treat, and $50 \%$ survival in those reaching surgery. Chromosomal anomalies, extracardiac malformations, and additional intracardiac malformations contributed to the presurgical and surgical mortality. This emphasises the importance of evaluating the karyotype, the extracardiac fetal anatomy, the pulmonary veins, the site and size of the atrial septal defect, the morphology of the tricuspid and pulmonary valves, and right ventricular function in a continuing pregnancy with the hypoplastic left heart syndrome. At the time of initial diagnosis of the hypoplastic left heart syndrome in a fetus in early pregnancy, the overall survival rate may be less than $40 \%$. In contrast, a full term neonate with ideal anatomy and without other anomalies may have a survival rate of over $70 \%$ at Norwood stage 1 . As additional complicating features are excluded, the prediction of risk can be modified progressively from the time of the initial diagnosis until the infant reaches surgery.

1 Allan LD, Sharland GK, Milburn A, et al. Prospective diagnosis of 1006 consecutive cases of congenital heart disease in the fetus. 7 Am Coll Cardiol 1994;23:1452-8.

2 Allan LD, Sharland GS, Tynan M. Natural history of hypoplastic left heart syndrome. Int $\mathcal{F}$ Cardiol 1989:25;341-3.

3 Allan LD, Cook A, Sullivan I, et al. Changing birth prevalence of the hypoplastic left heart syndrome as a result of fetal echocardiography. Lancet 1991;337:959-63.

4 Brenner JI. Prevalence and outcome of congenital heart disease in infancy: a 10 year population based experience. In: Wladimiroff JW, Pilu G, eds. Progress in obstetric and gynecological sonography series: Ultrasound and the fetal heart. New Yogical sonography series: Ultrasound and

5 Kern JH, Hayes CJ, Gersony WM, et al. Survival and risk factor analysis for the Norwood procedure for the hypoplastic left heart syndrome. Am f Cardiol 1997;80: $170-4$.

6 Mosca RS, Bove EL, Crowley DC, et al. Hemodynamic characteristics of neonates following first stage palliation for hypoplastic left heart syndrome. Circulation 1995; 92(suppl):II267-71.

7 Forbess JM, Cook N, Roth SJ, et al. Ten-year institutional experience with palliative surgery for hypoplastic left heart syndrome. Risk factors related to stage I mortality. Circulation 1995;92(suppl):II262-6.

8 Yagel S, Weissman A, Rotstein Z, et al. Congenital heart defects: Natural course and in utero development. Circulation 1997;96:550-5.

9 Ewigman BG, Crane JP, Frigoletto FD, et al, and the RADIUS study group. Effect of prenatal ultrasound screening on perinatal outcome. N Engl f Med 1993;329: $821-7$.

10 Saari-Kemppainen A, Karjolainen O, Ylostalo P, et al. Ultrasound screening and perinatal mortality: controlled trial of systematic one-stage screening in pregnancy. The Helsinki Ultrasound Trial. Lancet 1990;336:387-91.

11 Montana E, Khoury MJ, Cragan JD, et al. Trends and outcomes of prenatal diagnosis of congenital cardiac malformations by fetal echocardiography in a well defined birth population, Atlanta, Georgia, 1990-1994. I Am Coll population, Atlanta, Geor $1996 ; 28: 1805-9$.

12 Chang AC, Huhta JC, Yoon GY, et al. Diagnosis, transport and outcome in fetuses with left ventricular outflow obstruction. F Thorac Cardiovasc Surg 1991;102:841-6.

13 Sahn DJ, Rice MJ, Silverman NH, et al. Poor prognosis for fetuses with restrictive foramen ovale and left heart obstructive lesions: a multicenter study [abstract]. $7 \mathrm{Am}$ Coll Cardiol 1996;27:158A.

14 Razzouk AJ, Chinnock RE, Gundry SR, et al. Transplantation as a primary treatment for hypoplastic left heart tion as a primary treatment for hypoplastic left heart
syndrome: intermediate-term results. Ann Thorac Surg 1996;62:1-7. 